\title{
ILCEA
}

Revue de l'Institut des langues et cultures

d'Europe, Amérique, Afrique, Asie et Australie

$24 \mid 2015$

Lire et écrire ensemble

\section{Écrire ensemble. Un projet inachevé}

Write Together. An Unfinished Project

Escribir juntos. Un proyecto inacabado

Benoît Peeters

\section{OpenEdition}

Journals

Édition électronique

URL : http://journals.openedition.org/ilcea/3523

DOI : 10.4000/ilcea.3523

ISSN : 2101-0609

Éditeur

UGA Éditions/Université Grenoble Alpes

Édition imprimée

ISBN : 978-2-84310-313-1

ISSN : 1639-6073

Référence électronique

Benoît Peeters, «Écrire ensemble. Un projet inachevé », ILCEA [En ligne], 24 | 2015, mis en ligne le 03 novembre 2015, consulté le 19 avril 2019. URL : http://journals.openedition.org/ilcea/3523 ; DOI :

10.4000/ilcea.3523

Ce document a été généré automatiquement le 19 avril 2019.

(c) ILCEA 


\title{
Écrire ensemble. Un projet inachevé
}

\author{
Write Together. An Unfinished Project \\ Escribir juntos. Un proyecto inacabado
}

\section{Benoît Peeters}

1 Mes relations avec Michel Lafon ont très naturellement débuté sous le signe de Borges. C'est en effet à Cerisy-la-Salle, au colloque "Borges l'autre», que nous avons fait connaissance le 25 juillet 1981, dans le grenier du château. Plus de 33 ans sont passés. Je peux donc avouer que ce colloque fut la plupart du temps médiocre. Notre déception commune nous a rapprochés.

2 Très vite, l'écriture en collaboration est devenue l'un de nos sujets de conversation favoris. Nous étions frappés par le silence et presque le tabou qui entouraient cette pratique pourtant si répandue. Nous aimions beaucoup de celles et ceux qui avaient écrit ensemble : des sœurs Brontë aux frères Goncourt, de Dumas et Maquet à Hergé et Jacobs, de Boileau-Narcejac à Deleuze et Guattari.

Bientôt, c'est devenu un projet de livre. Des années durant, nous avons échangé des lettres et des notes, des esquisses et des plans. Le territoire s'élargissait, les références se multipliaient, de nouveaux dossiers s'ouvraient, comme si nous n'étions pas pressés d'aboutir. Nous est un autre, enquête sur les duos d'écrivains, le livre publié chez Flammarion en 2006 et longuement commenté dans l'entretien ici repris, n'est qu'un fragment de l'ample volume que nous avions imaginé.

4 Dans le second ouvrage, Écrire ensemble, on aurait retrouvé d'autres histoires de collaboration (Napoléon et ses " évangélistes », les frères Grimm et les Machado, Dickens et Collins, Dumas et son fils, Verne et le sien, Kipling et Balestrier, Fantômas, Conrad et Ford Madox Ford, Bourbaki, les Beatles, etc.). Mais globalement, ce livre aurait suivi un cheminement inverse du premier : les exemples devaient ici nourrir une tentative plus poussée de classification et de théorisation.

5 Ce second volume aurait notamment été l'occasion de dessiner une typologie de la collaboration : ses contextes d'apparition, son évolution et surtout ses méthodes. Mais nous nous serions aussi occupés d'étudier le passage entre écriture en collaboration et 
écriture collective, d'analyser les genres et registres privilégiés, les procédures spécifiques et les thèmes récurrents.

6 L'horizon de ces deux livres était de proposer une défense et illustration, en même temps qu'une théorie, de l'écriture en collaboration, mais aussi de voir ce que cette écriture hors norme, atypique, excessive, monstrueuse - en fin de compte moins mineure qu'on ne le dit, et peut-être même absolument moderne - nous enseigne de l'écriture individuelle.

7 J'ai retrouvé, à côté des esquisses de plusieurs chapitres, un sommaire assez précis de ce second volume. Je le reproduis ci-dessous. Sans Michel, je le sais, je n'écrirai jamais ce livre. Mais certaines des pistes que nous avions esquissées mériteraient sans doute d'être développées par d'autres chercheurs.

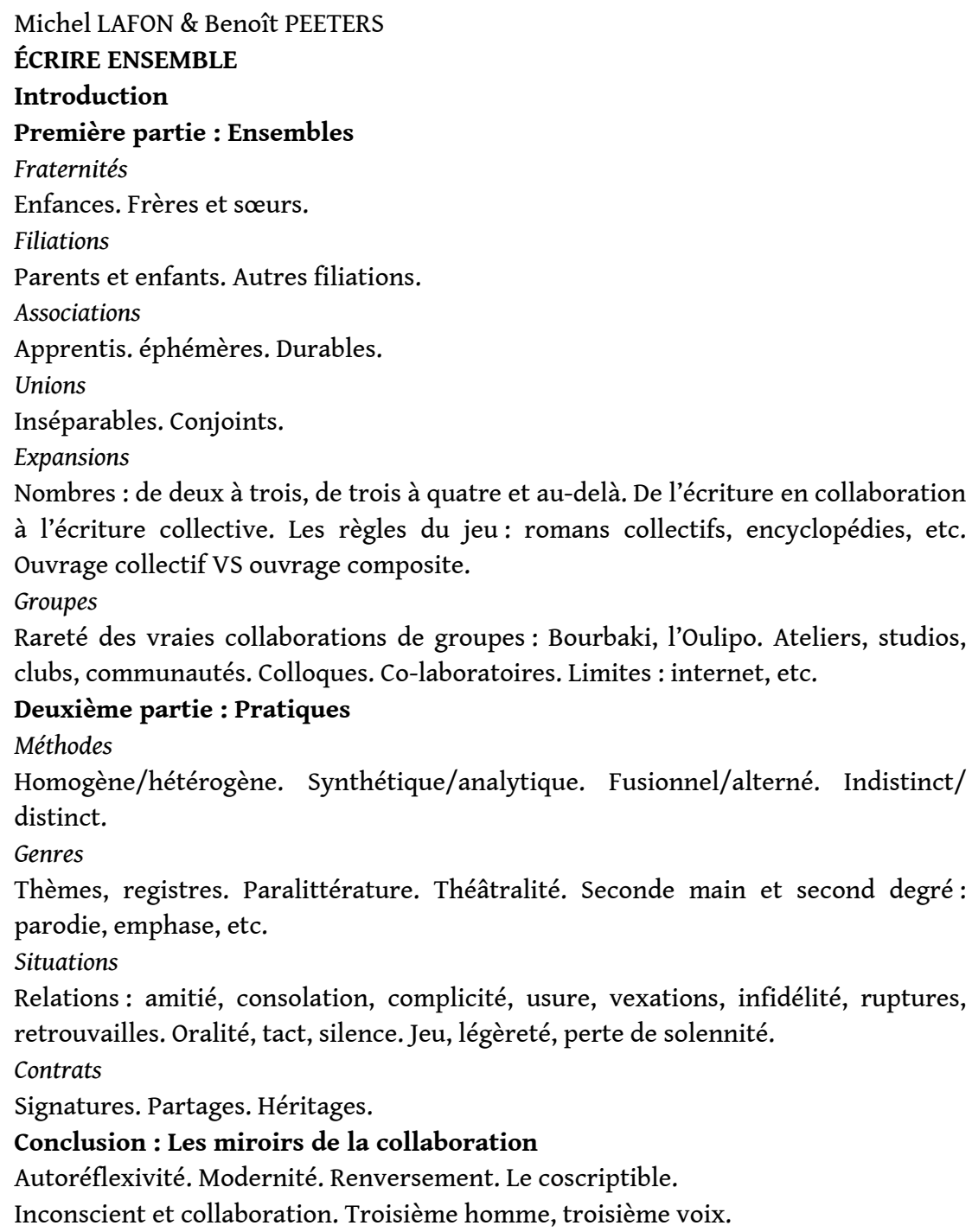

\section{Entretien avec Michel Lafon et Benoît Peeters}

\section{Qu'est-ce que la collaboration?}

La collaboration, étrangement, c'est d'abord un mot devenu à peu près imprononçable, dans l'emploi qui nous importe. Un mot encore confisqué, plus de soixante après, par l'histoire de la France sous l'Occupation allemande. Tout se passe comme si la censure 
pesant sur le phénomène littéraire était redoublée par cette étonnante censure lexicale. À preuve, nous avons renoncé, après bien des discussions entre nous et avec notre éditeur, à le faire figurer dans le titre de notre ouvrage, ce que nous regrettons d'ailleurs un peu aujourd'hui, tant il est vrai qu'il n'est pas mauvais de nommer clairement les choses dont on parle. Nous sommes contents de notre titre, Nous est un autre, mais nous le trouvons parfois un peu trop subtil, pas assez dénotatif... Tant qu'à écrire le premier ouvrage sur ce sujet, il n'aurait sans doute pas été inutile d'afficher clairement, d'emblée, la notion d'écriture en collaboration.

Vous proposez « dix-sept biographies » sur l'écriture en collaboration. Etait-ce là le parti pris initial du projet?

Au départ - c'est-à-dire il y a une bonne quinzaine d'années, lorsque, déjà amis, nous nous sommes rendu compte que nous avions tous les deux envie d'analyser cette pratique, que l'un a proposé à l'autre d'écrire ensemble le livre entrevu, et que l'autre a accepté cette proposition avec enthousiasme -, notre projet était de construire un ouvrage en deux grandes parties : une série d'études, puis une reprise théorique. La singularité des cas était si frappante, chaque histoire de collaboration si originale, si romanesque, chaque duo si attachant, qu'il nous aurait semblé dommage de dissoudre d'emblée ces cas dans un exposé systématique, en des centaines ou des milliers de biographèmes. Il y avait en outre une vertu pédagogique à dessiner d'abord un paysage, à embrasser l'horizon d'une pratique, puis à en tenter la théorisation, d'autant plus, on n'y insistera jamais assez, qu'il s'agissait d'un sujet absolument neuf : aucun ouvrage, à notre connaissance, n'a jamais entrepris l'étude systématique de l'écriture en collaboration.

Chemin faisant, nous nous sommes aperçus que le livre projeté était gigantesque (un bon millier de pages, vu le foisonnement de notre corpus et de nos pistes théoriques) et nous prendrait encore des années: nous nous enfoncions de plus en plus dans les recherches, le territoire de nos explorations ne cessait de s'agrandir. Même si nous gardons la nostalgie de ce livre total, il nous a semblé plus raisonnable d'entériner une première étape, de fixer, en quelque sorte, ce paysage. Nous avons donc choisi, sans grande difficulté, les cas qui excitaient le plus notre curiosité, ceux sur lesquels nous avions le plus de choses à dire, et qui bien sûr nous permettaient de traverser le plus grand spectre possible de pratiques (duo figé, duo évolutif, duo tournant, duo fusionnel, duo à distance, faux duo, atelier...) et de champs (fiction, mais aussi poésie, philosophie, psychanalyse, jusqu'au cinéma et à la bande dessinée) : il s'en est trouvé dix-sept, qui faisaient déjà un volume respectable. Et nous avons donc remis à plus tard l'exposé théorique que Nous est un autre promet et laisse régulièrement entrevoir.

N'existe-t-il aucun « écrire ensemble » au sens large ? Pourquoi avoir limité votre enquête aux duos d'écrivains (mis à part l'exception de l'atelier d'Hergé)?

Précisément, ce second ouvrage, annoncé dans notre introduction, s'intitulera Écrire ensemble, et il ne se contentera pas de proposer de manière systématique cette fois, et première par rapport aux exemples, la théorie des pratiques longuement exposées dans Nous est un autre; il s'intéressera aussi aux collaborations élargies, qui sont tout aussi foisonnantes, dans le corpus que nous avons accumulé, que les duos. Avec ce sentiment - ce pressentiment - que quelque chose de décisif a lieu quand on passe de deux à trois collaborateurs, ou de trois à quatre, ou a fortiori à des groupes bien plus nombreux: un saut qualitatif majeur, que nous sommes impatients d'analyser en 
profondeur, mais sur lequel nous avons déjà réuni beaucoup d'illustrations, et construit beaucoup d'hypothèses...

Estimez-vous que la notion d'un seuil entre influence, conseil, et véritable collaboration est possible? Peut-on parler, d'après vous, de « frontières de la collaboration »? Et dans le cas de la reprise par un tiers de l'œuvre d'un auteur, peut-on parler d'une forme ultime de collaboration?

Face à un sujet aussi neuf, qui apparaît bien comme un tabou traversant toute la littérature à l'âge moderne, il aurait été absurde, répétons-le, de ne pas limiter un corpus déjà proliférant, de ne pas circonscrire aussi rigoureusement que possible notre enquête. L'influence, la reprise, la continuation, et encore toutes les formes d'hypertextualité étudiées par Genette dans Palimpsestes, voire l'ensemble des catégories de la transtextualité, telle qu'il la définit dans les premières pages de cet ouvrage fondamental et fondateur, tout cela nous aurait amenés à voir la collaboration partout, et donc nulle part; à dissoudre un objet original dans une myriade de pratiques qui constituent, au fond, la littérature universelle : toute écriture, si l'on veut, est une écriture « en collaboration »; tout livre, toute littérature « collaborent » avec tous les livres et toutes les littératures... La fameuse formule de Montaigne, « Nous ne faisons que nous entregloser ", qui sert d'exergue, comme vous vous en souvenez sans doute, à un autre ouvrage important, contemporain de Palimpsestes, La Seconde Main ou le Travail de la citation, d'Antoine Compagnon, suggère aisément un « Nous ne faisons que collaborer $" . .$.

Il importe donc, selon nous, de réserver le terme à des pratiques répondant à un minimum de caractéristiques communes: l'écriture en collaboration relève d'une volonté commune et débouche, via un travail en commun, sur un produit commun. Autrement dit, on collabore avec un contemporain; on ne collabore pas avec lui à son insu, mais en conscience, sinon en coprésence, en vertu d'un contrat, implicite ou explicite, formalisé ou latent; et cette pratique commune ( $c$ commune» mais qui mobilise des processus si variés, si inattendus, si changeants que nous préférons ne pas les classer ou les énumérer dans le cadre de cet entretien : nous renvoyons à nos dixsept cas, et aux quelques grandes figures fondamentales de la collaboration qu'ils laissent apparaître...), cette pratique commune, disions-nous, débouche sur un objet commun, un texte commun s'il s'agit de littérature, qui peut rester anonyme, porter la signature des deux (dans le cas d'un duo), ou la signature d'un seul, ou un pseudonyme, ou toute autre combinaison : la littérature, on en a la confirmation chaque fois que l'on mène ce genre d'enquête, est toujours plus inventive que le plus inventif des poéticiens!

On le comprend donc: lorsque deux auteurs créent au voisinage l'un de l'autre, sous le regard l'un de l'autre, en osmose permanente, mais qu'ils produisent deux œuvres distinctes, et non un texte unique, nous ne parlerons pas de collaboration : des œuvres croisées ne sont pas une œuvre en collaboration.

Mais aussitôt posées, de telles évidences se lézardent : il existe en effet des cas où une collaboration intime, continue, absolue, produit deux textes distincts, deux œuvres distinctes, et pourtant indissociables; des cas où ces œuvres distinctes portent la marque de leur gestation commune, la trace de regards croisés, de pratiques interpénétrées. Comme il existe aussi des cas où un créateur unique, et même solitaire, invente une collaboration fictive, à laquelle il peut parvenir à donner corps (on aura reconnu, bien sûr, le cas Gary-Ajar-Pavlovitch). D'où l'intérêt pour nous de toucher, comme vous le suggérez, aux frontières de la collaboration : c'est parfois dans ces cas 
limites, qui sont encore, d'une manière ou d'une autre, fût-ce imaginaire, fantasmée, des cas de collaboration, que nous avons appris les choses les plus fortes sur cette pratique.

Est-ce par souci de maintenir une certaine unité dans la notion de collaboration que vous avez écarté, non sans les mentionner dans l'introduction, les autres domaines de la création artistique? S'agissait-il pour vous d'aller voir seulement « là où ça fait mal » ?

Nous avons quand même consacré un chapitre au cinéma (Prévert et Carné), et un chapitre à la bande dessinée (Hergé \& Cie) ; mais il est vrai que les domaines artistiques sont minoritaires dans notre table des matières, largement « littéraire » (au sens large, incluant fiction et diction, si vous voulez). Mais pourquoi supposer que la collaboration littéraire ferait "plus mal» que tout autre type de collaboration artistique, voire scientifique, etc.? Le «co-laboratoire» des scientifiques est-il jonché de pétales de roses? Les ateliers d'artistes sont-ils toujours paisibles? Les Beatles et d'autres groupes fameux ne se sont-ils pas rapidement séparés? Les questions de droits d'auteurs, de brevets, de propriété intellectuelle ne se posent-elles pas dans ces lieux de façon aiguë ? Nous avons choisi la littérature parce que dans son champ la collaboration est l'exception, à l'âge moderne, ou en tout cas parce qu'elle est pensée comme l'exception, jusqu'au déni de réalité parfois (alors que, dans bien des arts, elle est la règle...). Bref, plutôt que d'aller voir, comme vous dites, « là où ça fait mal », il s'agit plutôt pour nous d'aller voir « là où ça ne se fait pas ", ou bien "là où ça se fait rarement ", en tout cas « là où il semble que ça ne se fasse pas »...

Le travail de traduction peut-il à son tour être appréhendé comme une forme de collaboration? Michel Lafon, pourriez-vous éventuellement fournir un témoignage direct à ce propos, par rapport à votre expérience personnelle de traducteur?

Toute traduction n'est pas collaboration; on ne collabore pas avec Cervantès, quand on traduit le Quichotte (il est plus facile, dirait Pierre Ménard, d'être Cervantès que de collaborer avec lui, ou de le réécrire, ou de le traduire!). Mais traduire un écrivain contemporain, avec qui l'on est en relation, que l'on peut interroger, oui : rien n'empêche de considérer que la traduction finale, le «texte d'arrivée » est le produit d'une collaboration entre l'auteur et le traducteur, voire entre l'auteur $n^{\circ} 1$ et l'auteur $\mathrm{n}^{\circ} 2$. Toutes les conditions sont bien remplies pour l'attester (contemporanéité, intentionnalité, contrat, unicité...). Une collaboration qui peut être évidemment très déséquilibrée, la part respective de chacun des deux pouvant être très inégale, comme elle peut l'être d'ailleurs dans tout autre type de collaboration, ce qui n'invalide en rien la réalité (ni la qualité) d'une collaboration.

On peut s'amuser à envisager des cas plus riches, voire plus retors, de collaboration entre auteur et traducteur. C'est Harry Matthews traduisant Georges Perec, et Georges Perec traduisant Harry Matthews (comme César Aira, l'écrivain argentin, traduisant en espagnol Nous est un autre ${ }^{1}$, dont un des deux co-auteurs a traduit onze de ses romans en français) : autrement dit, auteur et traducteur échangeant les rôles et entrant chacun dans l'intimité textuelle de l'autre, en parfaite ou en relative réciprocité.

Un autre cas, pas si inhabituel que ça : le traducteur signale à l'auteur une incohérence, en tout cas une obscurité, une zone d'incompréhension. L'auteur constate alors que son original est fautif, ou du moins qu'il ne le satisfait plus, il demande à son traducteur de rectifier, d'améliorer - plutôt que de le faire lui-même. Si son ouvrage est réédité dans sa langue originale, il portera éventuellement la trace de cette correction seconde, ce qui est une belle marque de collaboration à rebondissement, vous en conviendrez ! 
Un autre cas encore, sans doute plus exceptionnel : la relation est suffisamment étroite entre les deux pour que l'auteur parle dans son roman de son traducteur, en fasse un de ses personnages, utilise ses souvenirs, ses confidences, le mette en scène, reproduise ses phrases au mot près, cite tel passage de sa correspondance, utilise telle page de son œuvre, ou même lui consacre un livre tout entier, sous son nom ou sous quelque masque. Ici, c'est dès l'original, avant même que le traducteur s'empare du texte pour le traduire, que la collaboration a lieu, c'est le « texte de départ » qui est le produit d'une collaboration implicite, voire clandestine entre auteur et traducteur (tout cela peut s'opérer à l'insu du traducteur, qui ne le découvre que le jour où il reçoit et lit le livre de son ami), ou plutôt entre l'auteur et cette personne à qui le lie un rapport privilégié, et qui se trouve par hasard être aussi son traducteur. Au moment où ce traducteur commence à traduire un tel texte (si tant est qu'il se sente capable et, surtout, désireux de traduire un texte aussi étrangement produit, qui l'interpelle, l'implique et le « dérange » à ce point), il va en quelque sorte collaborer avec l'auteur et avec lui-même en tant que personnage, qu'inspirateur momentané, que double de l'auteur, retrouver au moins par endroits ses propres mots sous les mots de l'« original», ses propres tics de langage, sa propre mémoire, sa propre bibliothèque... Traduire, au moins pour certaines de ces pages originales, consistera alors à revenir à soi plus qu'à aller vers l'autre, en un étonnant raccourci de soi à soi qui pourrait tendre à nier l'auteur officiel et à instaurer son double inspirateur et traducteur en instance auctoriale supérieure, puisqu'elle est à la fois, en quelque sorte, au début et à la fin du processus d'écriture... Vous voyez que lorsque nous disons que la collaboration est en avance sur toute théorisation a priori, sur toute taxonomie préalable, nous n'exagérons pas !

Vous évoquez en introduction de l'ouvrage cet «étrange tabou » qui traverse l'histoire de l'écriture en collaboration. Dans quel parti pris idéologique entendez-vous ce tabou ? S'agitil d'une difficulté à abandonner le paradigme romantique du créateur comme génie solitaire?

Oui, bien sûr, il s'agit du lien persistant au paradigme romantique de l'auteur unique, dont même la "modernité" s'est moins délivrée qu'elle ne l'a cru. L'idée de collaboration continue de gêner la plupart des écrivains, y compris les plus novateurs. En écrivant à deux, croient-ils souvent, on déchoirait de manière quasi automatique, tombant de la littérature à la "paralittérature ». Le plus étrange, c'est que cette idée reçue a contaminé jusqu'à des auteurs ayant pratiqué, de façon remarquable, l'écriture en collaboration. La très injuste condescendance avec laquelle on considère un peu partout l'œuvre signée Bustos Domecq a été encouragée par l'attitude de Borges et de Bioy Casares eux-mêmes.

Les enjeux complexes que pose la notion de collaboration ne dérivent-ils pas de la collaboration elle-même, des démarches créatrices singulières qu'elle suppose?

Notre travail repose en effet sur une intuition, que chaque étude n'a fait que fortifier : la collaboration est une démarche singulière, atypique, aberrante, hors norme (dont on pourrait dire que le "produit commun" auquel elle aboutit est par essence un "produit absolument hors du commun »), en tout cas une démarche qui implique une théorie singulière, spécifique, une théorie qui est de nature à révolutionner, ou du moins à relancer les théories littéraires fondées tacitement sur l'observation, l'analyse de l'écriture individuelle.

Dans I'histoire de cette notion, les cas les plus connus sont malheureusement ceux qui sont passés devant les tribunaux, ou ont donné lieu à des négociations parfois âpres sur 
les revenus de chacun. Pourrait-on dire que la collaboration ne se révèle que lorsqu'elle échoue plus ou moins?

Il est vrai que beaucoup d'histoires de collaborations célèbres finissent mal ; mais c'est souvent l'indice qu'elles ont duré longtemps, ce qui n'est pas rien. Certains à-côtés de la collaboration, certaines pulsions d'amour et de haine se révèlent en effet devant les tribunaux, ou du moins dans des rancœurs tardives. Mais après tout, quand un duo non collaboratif tourne mal, quand un couple se délite, cela n'enlève rien à ce qui a pu se passer entre ses membres - a fortiori si une œuvre a été produite. Une collaboration qui se termine dans les procès, dans les drames ou les récriminations n'est pas forcément une collaboration qui échoue: c'est simplement une collaboration qui s'achève. N'est-ce pas dans leurs œuvres communes (des Trois Mousquetaires à Ingénue, en passant par Le Comte de Monte-Cristo ou Le Vicomte de Bragelonne) que Maquet et Dumas ont révélé le meilleur de leurs talents respectifs? Une collaboration, dont, pardelà tous les litiges, ils garderont l'un et l'autre, jusqu'au bout, la plus intense nostalgie...

Globalement, davantage que dans les doléances et les dénonciations, c'est dans la spécificité et la profondeur d'un texte écrit à quatre mains que nous avons trouvé les traces les plus subtiles, les plus retorses, les plus riches de la collaboration dont il est issu. Les textes produits en collaboration nous en disent plus que les procès en justice sur leur processus d'écriture. Concrètement, nous avons ainsi découvert que les fictions produites en collaboration racontent très souvent - pour ne pas dire toujours -, d'une manière ou d'une autre, plus ou moins cryptée, métaphorique, etc., des histoires de collaboration! C'est pour nous un des enseignements majeurs de cette « enquête sur les duos d'écrivains ».

Une collaboration évidente pour nous reste l'ouvrage collectif (actes de colloques, numéro spécial de revue). La mention de chacun des acteurs du projet, de son rôle spécifique à l'intérieur de ce projet (auteur, traducteur, éditeur), marque qu'il ne s'agit là que d'une pensée, d'une écriture mise en présence, en confrontation. Peut-on néanmoins tenter de situer pareilles entreprises dans l'ombre portée de la collaboration?

C'est en effet un des cas les plus fréquents de l'écrire ensemble, bien au-delà du duo, un de ces cas auxquels nous voulons consacrer notre prochain livre en commun. S'agit-il ou non de collaboration? Cela revient surtout à poser la question de la visibilité des contributions de chacun (signatures, interventions délimitées, rôles répartis). Or, dans les collaborations littéraires à deux, il n'y a pas forcément indistinction, fusion et synthèse : pensons à Par les champs et par les grèves de Flaubert et Du Camp, dont chaque contribution est clairement délimitée et identifiable; mais aussi à toutes les fictions écrites sur le mode de l'alternance : A rédige le début, puis passe le relais à $\mathrm{B}$ qui rédige la suite, puis qui le repasse à $A$, et ainsi de suite; pour peu que l'on connaisse l'ordre de passage et le moment où il a lieu, on n'est plus si loin, au moins en structure, de l'ouvrage collectif de type scientifique, universitaire, etc.

Sans remonter à L'Encyclopédie de Diderot, d'Alembert et al., les Éléments d'histoire des sciences, ouvrage collectif dirigé par Michel Serres, sont un bon exemple d'un ouvrage scientifique pluriel qui va au-delà de la simple mise bout à bout de contributions, en tentant véritablement le pari de la collaboration intellectuelle: réunions, confrontations et échanges à différentes étapes, tels que les expose l'introduction du volume. Le livre collectif de type universitaire peut sans doute trouver dans une élaboration de ce type (progressive, évolutive et réflexive) les meilleures chances de se renouveler et, partant, de se perpétuer. Qui lit encore, de nos jours, un de ces numéros 
de revue universitaire " à thème ", dont le disparate, l'hétérogène, l'aspect attrape-tout sautent trop visiblement aux yeux...? On est arrivé à une époque où il faut réinventer ce type de pratique, y compris et peut-être d'abord à l'Université! Internet, et notamment un projet encyclopédique comme Wikipedia, jouent à cet égard un rôle évident d'accélérateur.

L'horizon de la collaboration, vous le soulignez à plusieurs reprises, reste de parvenir à une voix sans origine (que l'on en parle à la Barthes) ou bien encore à un «réagencement énonciatif» indémêlable (que l'on reprenne les concepts deleuziens). Aussi, de ces collaborations-là, lorsqu'elles réussissent, que reste-t-il à dire? Ne touche-t-on pas là à quelque chose qu'approchait déjà Foucault, et à sa formule écrire pour n'avoir plus de visage $?$

De ces collaborations réussies, il reste précisément tout à dire ; elles sont à l'horizon de la révolution littéraire (philosophique, poétique, etc.) qui est pour nous indissociable de cette pratique inouie, et en tout cas si rarement interrogée.

À bien y réfléchir, même si c'est un cas limite, le cas Gary-Ajar-Pavlovitch représente plutôt une apothéose, ou une hypertrophie de la fonction auctoriale, qu'un début de disparition. Étrangement, aujourd'hui, c'est parce qu'il s'est fait autre et a disparu en tant qu'écrivain trop identifié que Romain Gary s'est sauvé en tant qu'Auteur. Et que dire de l'ego infatué d'un Bustos Domecq, le troisième homme né de la fusion d'Adolfo Bioy Casares et de Jorge Luis Borges et prenant, dès son surgissement et pendant quarante ans, le pouvoir sur eux, en même temps qu'il tient ferme leur plume? Avec cette figure caricaturale, excessive, délirante de l'Auteur, se réinventant lui-même et multipliant à plaisir les instances qui l'autorisent, électrisant la littérature argentine et peut-être toute la littérature latino-américaine, bouleversant ces littératures avec une puissance révolutionnaire, on assiste à une réactivation violente du sujet et des sujets - et l'on est au plus loin, comme vous le voyez, du thème de la «mort de l'Auteur " cher à Blanchot, à Foucault ou à Barthes !

D'après les auteurs que vous avez étudiés et dans votre propre pratique scripturale, la création en collaboration génèrerait-elle plus de censure, ou au contraire, ferait-elle en sorte que les censures s'abolissent?

Chez les auteurs que nous avons étudiés, la collaboration nous est apparue presque systématiquement comme le lieu de l'abolition des censures personnelles, l'occasion idéale pour le surgissement d'une liberté nouvelle, révolutionnaire, fracassante. Sur un mode mineur ou sur un mode majeur (les multiplicités de Deleuze et Guattari, le troisième homme de Borges et Bioy, « l'homme coupé en deux » de Breton et Soupault... ), tous nos cas racontent l'histoire d'une libération, d'un au-delà des limites personnelles, de l'invention d'une manière radicalement autre de créer. C'est pour nous l'aspect le plus passionnant de la création en collaboration, celui qui contredit le plus fondamentalement les lieux communs qui caricaturent cette pratique, et en détournent le regard, jusqu'au déni et au tabou.

Si nous évoquons maintenant notre cas personnel, il nous faudrait toutefois nuancer : la collaboration a surtout été pour nous une exigence supplémentaire de rigueur et d'inventivité (écrire un chapitre pour surprendre l'autre, pour l'« épater » si possible, dans l'attente d'une relance encore plus surprenante et inventive). Dans une amitié constante, une estime profonde sans laquelle nous n'aurions pas écrit une seule page de ce livre : on ne collabore bien qu'avec quelqu'un que l'on admire, d'une façon ou d'une autre, quelqu'un dont on attend un enrichissement (pourquoi perdre mon temps avec un autre, si j'en sais plus que lui, s'il ne m'apporte rien?), et avec qui, s'il nous est 
permis de l'affirmer au moins pour notre propre cas, rien ne se joue de l'ordre de la névrose. C'est à coup sûr le cas des collaborations les plus durables: Bioy et Borges, exemplairement; mais aussi, pendant l'essentiel de leur relation (ici encore, la fin ne doit pas occulter le long déroulement), Erckmann et Chatrian, Prévert et Carné ou Boileau et Narcejac...

Cela étant posé, quand nous faisons le bilan de notre premier ouvrage commun, il nous arrive de nous dire que la collaboration nous a considérablement ralentis (Ralentir travaux: le titre du petit ouvrage poétique collectif d'André Breton, René Char et Paul Éluard nous fait un bel emblème!). Peut-être parce que, pendant des années, nous avons écrit davantage l'un pour l'autre (pour ce premier lecteur obligé qu'est l'autre membre du duo), que pour le lecteur virtuel, le lecteur à venir... Mais nous ajoutons aussitôt : écrit par l'un de nous deux seul, le livre aurait paru quelques années plus tôt, mais il aurait été incomparablement moins varié, moins complet et moins riche, certains de ses chapitres représentant parfois des années de travail, pour ne pas dire toute une vie de réflexion (du moins d'imprégnation), de l'un ou de l'autre, voire des deux parallèlement... Et toujours, évidemment, dans l'échange le plus total : qui sait, par exemple, si la plus belle idée du chapitre sur Hergé ne vient pas du tintinologue le plus secret de nous deux, et si la plus belle idée du chapitre sur Bioy et Borges ne vient pas du lecteur de littérature argentine le moins reconnu de nous deux ?

Je pense à l'envoi qui termine votre ouvrage (cette biographie fictive de votre propre collaboration), qui s'autorise plus de sensualité et de fiction. Ce pas fictionnel, qui surprend dans le cadre d'un ouvrage critique, aurait-il été possible sans collaboration, ou bien est-ce la configuration énonciative singulière de l'écriture à quatre mains qui l'a rendu possible?

C'est exactement ça : la collaboration a favorisé le surgissement de cette fiction finale. Nous nous sommes retrouvés une dernière fois pour écrire ensemble cette fin et, pendant les semaines précédant ce rendez-vous, chacun de son côté a essayé de rédiger une ébauche de conclusion « sérieuse ", puisque notre ambition était, dans ces ultimes pages, d'esquisser la théorie suscitée par la diversité des dix-sept cas, en attente d'une exposition détaillée dans notre deuxième volume. Nous n'avancions pas, jusqu'à ce que, la veille de nos retrouvailles, l'un de nous lance : «Et si on le faisait sous la forme d'une fiction? » Il nous est vite apparu que c'était la bonne solution, et même la solution idéale, et qu'elle ne pouvait venir que de notre entre-deux. Nous avons écrit ces pages très vite, avec jubilation et facilité, dans une espèce d'euphorie. Non seulement la fiction s'est avérée un mode idéal de fonctionnement, à ce stade-là de notre livre, mais surtout elle nous a permis de dire en huit pages une quantité de choses (de tirer les leçons théoriques de nos cas, et même d'évoquer d'autres hypothèses théoriques, débordant lesdits cas) dont l'exposition « sérieuse » aurait pris cinquante pages! Cela nous a d'ailleurs donné, à l'un et l'autre, une furieuse envie d'écrire ensemble un roman.

Une « poétique » de la collaboration est-elle réalisable? Ou bien toute collaboration réussie suppose-t-elle une indistinction qui rend caduque la volonté d'étude?

Oui, il ressort de tout cela qu'une poétique est possible, et même qu'il était urgent d'en proposer une : c'est ce que nos dix-sept «récits critiques » s'efforcent de faire, d'une manière aussi narrative, aussi plaisante, aussi lisible que possible.

Attention toutefois : parmi les à priori qui, à notre sens, peuvent fausser la recherche, il y a celui d'un idéal de collaboration comme fusion. La fusion n'est qu'un cas de la collaboration, il est risqué d'en faire l'horizon de toute collaboration, la garantie d'une 
collaboration "réussie». On pourrait dire la même chose de l'équilibre des contributions, ou de l'égalité des tâches. Le déséquilibre (réel ou apparent) des contributions, la hiérarchisation (apparente ou réelle) des tâches ne sont pas forcément des défauts, ni le symptôme d'un inaboutissement, encore moins la preuve d'une injustice ou d'une mésentente. Idem pour l'indistinction: certaines collaborations gagnent sans doute à y parvenir, d'autres ménagent la distinction claire et nette des contributions et des contributeurs, et ne sont pas pour autant moins abouties... Il reste de ce côté de très nombreuses « figures » à étudier.

D'après vous, dans une étude sur le travail en collaboration, que peut éclairer la correspondance? Est-elle le lieu scriptural où se dévoilent ces relations d'intimité dont vous avez à chaque fois retracé la biographie? Le lieu où se constitue le mythe de cette collaboration?

Dans de nombreux cas, surtout avant l'invention des modes de communication actuels, la correspondance est le vecteur irremplaçable d'une collaboration qui ne s'opère pas en coprésence des duettistes (Edmond et Jules de Goncourt n'ont pas à s'écrire, évidemment, puisqu'ils ne se sépareront que deux fois dans leur vie siamoise !) : qu'il s'agisse des billets que Dumas envoie plusieurs fois par jour à Maquet, pour le presser de lui envoyer sa copie, ou des télégrammes ludiques échangés par Boileau et Narcejac ( Essayons le poison »), ils constituent des sources magnifiques pour une telle étude. Mais il y a plus : dans le cas de la correspondance d'Erckmann et Chatrian, par exemple, on est au cœur même de la collaboration. Dans le dispositif très étrange, très intime, très déséquilibré et pourtant très durable et très productif, sans doute très névrotique aussi, qu'ils ont éprouvé la nécessité de mettre en place tôt dans leur vie, leurs lettres sont sans doute le lieu où se joue la scène même de la collaboration.

En ce sens, croyez-vous que l'apport d'une étude génétique complèterait votre étude? Serait-elle un biais judicieux par lequel prendre la question que vous avez explorée tout au long de votre ouvrage?

Oui, mais à condition, nous y insistons, de ne pas partir d'à priori théoriques du type de ceux que nous venons de dénoncer. La lettre et le manuscrit ne révèlent pas tout. Il est passionnant, et même émouvant, de voir deux graphies alterner, se corriger, se reprendre, ou au contraire une seule graphie s'étaler, au nom d'un duo. Mais l'écriture peut n'être qu'un geste, une simple trace : écrire une idée n'est pas forcément avoir eu cette idée ; écrire un mot n'est pas forcément avoir trouvé ce mot; qui écrit n'est pas forcément qui pense ni qui parle, etc. Il convient d'envisager toutes les figures de l'écrivain, de l'écrivant, du scribe, du secrétaire, du copiste... Comment ne pas évoquer encore la plus intime de toutes les collaborations, la plus monstrueuse, la plus fascinante, celle des frères Goncourt? L'exemple de leurs manuscrits - où, sur d'assez longues périodes, un seul et même frère tient la plume, généralement Jules - suffirait à montrer cette complexité : une seule main pour deux cerveaux, une seule graphie pour une combinaison de fantasmes gémellaires, aux limites de la psychose... Bref, à aucune étape de l'étude de l'écriture en collaboration il ne convient, à notre avis, de se focaliser sur la question du "qui a écrit quoi ?». C'est le piège que tend la collaboration à la critique, et exemplairement, donc, à la critique génétique, c'est aussi le piège qu'elle tend à la théorie, c'est la pente facile et gratuite du lieu commun. La collaboration est précisément cette pratique instable, rebondissante, explosive où je peux pousser la délicatesse, la complicité ou la perversité jusqu'à proposer à l'autre, pour la page commune, une séquence qui semble issue directement de lui, en espérant que l'autre, 
en retour, me proposera une séquence qui paraisse sortie directement de ma bouche, ou de ma plume...

\section{NOTES}

1. L'ouvrage est paru en 2008 chez l'éditeur argentin Beatriz Viterbo, sous le titre Escribir en colaboración - Historias de dúos de escritores.

\section{RÉSUMÉS}

À quatre mains, Benoît Peeters et Michel Lafon ont écrit le livre de référence sur l'écriture en collaboration. Nous est un autre, enquête sur les duos d'écrivains (Flammarion, 2006) a marqué un tournant dans la réflexion sur la création littéraire, sur la notion d'auteur et plus amplement sur le fait littéraire.

Benoît Peeters reprend ici en quelques paragraphes l'histoire d'une longue amitié mais surtout celle d'une écriture in progress, en dévoilant le contenu du second volume de leur projet, plus théorique, qui ne verra malheureusement pas le jour. Ces lignes sont également une introduction à un entretien à deux voix que Michel Lafon appréciait particulièrement.

For four hands, Benoît Peeters and Michel Lafon wrote the reference book on the collaborative writing. Nous est un autre, enquête sur les duos d'écrivains (Flammarion, 2006) marked a turning point in thinking on literary creation, on author's notion and more fully on the literary fact.

Benoît Peeters gets here in some paragraphs the story of a long friendship but especially that of one writing in progress, by revealing the contents of the second volume of their project, more theoretical, which will not unfortunately be born. These lines are also an introduction to an interview which Michel Lafon particularly fond.

A cuatro manos, Benoit Peeters y Michel Lafon escribieron el libro de referencia sobre la escritura en colaboración. Escribir en colaboración, historias de dúos de escritores (Viterbo, 2008) marcó un giro en la reflexión sobre la creación literaria, sobre la noción de autor y de manera más amplia sobre el hecho literario.

Benoît Peeters recuerda en estos párrafos la historia de una larga amistad pero sobre todo la de una escritura in progress, entregando el cotenido del segundo volúmen de su proyecto, más teórico, que lamentablemente no saldrá a la luz. Estas líneas sirven también de introducción a una entrevista a dos voces que Michel Lafon apreciaba particularmente. 
INDEX

Mots-clés : écriture en collaboration, duo d'écrivains, théorie littéraire, notion d'auteur

Palabras claves : escritura en colaboración, dúo de escritores, teoría literaria, noción de autor

Keywords : writing in collaboration, duet of writers, literary theory, author's notion 